\title{
CULTURAL AND MORPHOLOGICAL VARIABILITY OF ALTERNARIA LINI ISOLATES CAUSING BLIGHT OF LINSEED.
}

\section{A.K.VERMA \& R.B.SINGH}

Department of Plant Pathology N.D. University of Agricultural \&Technology Kumarganj, Faizabad- (U.P.) India

\begin{abstract}
Alternaria lini causes blight of linseed in India. The leaves showing typical symptoms of Alternaria blight, in linseed collected from different part of country and characterized for cultural, morphological, pathogenic and molecular variations. Alternaria lini colonies varied in respect of their colony colour (whitish, light brown to dark brown), growth pattern (slow, medium to fast), appearance (cottony, fluffy, feathery to compressed \& thin), radial growth (25mm to 60mm), shape (circular), margin (wavy, rough to smooth), zonation (presence or absence), and sporulation (early, medium to late). Morphological variation in average conidial length (23.26 to 45.72 $\mu \mathrm{m})$, width (6.76 to $17.01 \mu \mathrm{m})$, number of septa (1.86 to 5.60) and presence or absence of beak (unbeaked, rudimentary beaked and short beaked) were observed. Average conidial size ranged between $24 x 7$ to $46 x 17 \mu m$.

KEYWORDS: Alternarialini, Linum, Cultural and Morphological Variability
\end{abstract}

Received: Nov 06, 2017; Accepted: Nov 26, 2017; Published: Dec 02, 2017; Paper Id.: IJASRDEC201749

\section{INTRODUCTION}

Linseed (Linumusitatissimum L.), belongs to the family Linaceae and the genus Linum is commonly known as "Ulsee" or "Tisee". It contains 20 to 30\% protein. Feeding linseed and its oil may be medicated in hyperlipidemia, to reduce and control arteriosclerosis, thrombosis and mycocardial infraction, by reducing cholesterol and low density lipid (Vajpeyiet al., 2005). Linseed oil is a triglyceride, like other fats. Linseed oil is distinctive for its unusually large amount of $\alpha$-linolenic acid, which has a distinctive reaction with oxygen in air. Specifically, the fatty acids in a typical linseed oil are the tri -polyunsaturated $\alpha$-linolenic acid (51.9-55.2\%), the saturated acids palmitic acid (about 7\%) and stearic acid (3.4-4.6\%), the monounsaturated oleic acid (18.5-22.6\%), the doubly unsaturated linoleic acid (14.2-17\%). Among oilseed crops grown in India during Rabi. Linseed is next in importance to rapeseed- mustard in acreage as well as in production. India stands at fourth position in global production (Anonymous, 2013). Linseed is known to suffer from many diseases such as blight, rust, canker, damping off, leaf spot, root rot and wilt (Kolte and Fitt, 1997). Alternaria blight, earlier designated as a minor disease has assumed greatest importance, in different parts of the country, especially mid eastern India. Alternaria blight caused by Alternaria lini Dey and A. linicola Grooves and Skolko, is a major biotic stress, limiting crop yield in hot and humid environment (Singh and Singh 2004, 2005).

Out of two species of Alternaria known to occur on this crop, Aternaria lini is a more severe one. Survey of literature revealed that no information is available on variability of A. lini or A. linicola causing blight of linseed in India or outside of country. Since still no true resistance is available against this disease, it indicates the presence of variability in pathogen in different agro climatic zone of the country. To develop the resistant cultivar, it 
requires stable resistant donor for this kind of variability in blight causing pathogen is necessary. Hence, present investigation was undertaken with pathogenic and molecular variability.

\section{MATERIALS AND METHOD}

The experiment was carried out in glass house, laboratories of Department of Plant Pathology and Plant Molecular Biology \& Genetic Engineering (PMB\&GE) of Narendra Deva University of Agriculture and Technology, Kumarganj, Faizabad (U. P.) during year 2012-13 and 2013-14. Samples were collected from different agro climatic zones of India (Patna, Bilaspur, Sultanpur, Mauranipur, Mashodha, Kanpur, Kangra, Ranchi, Ranchi, Mirzapur, Gorakhpur, Berhampur, Kumarganj and Jammu). Potato-Dextrose-Agar medium was used for maintaining of pure culture of Alternariaspecies. Different media viz., Radish agar medium, Maize meal medium, Barley meal medium, Linseed leaf extract medium, Carrot agar medium as well as Potato Dextrose Agar medium were used for cultural studies of the A. lini. Effect of different culture media on colony colour, appearance, pattern of growth, shape, margin, zonatoin, sporulation and radial growth of 12 isolates of Alternarialini on potato dextrose agar medium, radish agar medium, maize meal medium, barley meal medium, linseed leaves extract medium and carrot agar medium were observed from 7 days old culture. Morphological variability of 12 isolates were studied. The different characters viz. size of conidiophores and conidial characters such as length, width, colour, shape, and number of septa were studied on Potato Dextrose Agar (PDA) medium. The size of conidiophores and conidia were examined under microscope using ocular micrometry (Meenaet al. 2005). Visual observation for septation was done by using light microscope (40X) taking 20 conidia from each slide. Length range and average length, width range and average width, range of horizontal septa of conidia and conidiophores were taken.

\section{RESULTS AND DISCUSSIONS}

\section{Cultural variability}

Variability of 12 isolates collected from different places studied by culturing them on different media (Potato dextrose agar medium, Barley meal medium, Radish agar medium, Carrot agar medium, Maize meal medium, and Linseed leaf extract medium) to find out their growth pattern, colonies colour, appearance, shape, margin, zonation and sporulation. The results obtained from the studies are discussed as under:

On potato dextrose agar medium the colonies colours of different isolates were noted as whitish, dirty white, light brown and dark brown. Variation on colonies colour of different isolates of Alternaria solani were studied by Kumar et al. (2007) and Singh et al.(2014) in case of tomato and they also reported variable colonies colour, such as brownish black, greenish black, reddish black, whitish black, and yellowish. Pramila et al. (2014) worked on variability of Alternaria brassicae isolates of Uttarakhand and reported whitish to grayish colonies colours. The variation of colonies colours in present study, supports the finding of above workers.

Besides PDA, colonies of isolates grown on radish agar medium showed dirty white $\left(\mathrm{Al}_{10}\right)$, light brown $\left(\mathrm{Al}_{4}, \mathrm{Al}_{6}\right.$, $\mathrm{Al}_{7}, \mathrm{Al}_{8}, \mathrm{Al}_{9}, \mathrm{Al}_{11}$ and $\left.\mathrm{Al}_{12}\right)$ and brown $\left(\mathrm{Al}_{1}, \mathrm{Al}_{2}, \mathrm{Al}_{3}\right.$ and $\left.\mathrm{Al}_{5}\right)$ colours. Isolates also showed variation on maize meal medium as white $\left(\mathrm{Al}_{10}\right)$, dirty white $\left(\mathrm{Al}_{1}, \mathrm{Al}_{2}\right.$, and $\left.\mathrm{Al}_{5}\right)$ and light brown $\left(\mathrm{Al}_{3}, \mathrm{Al}_{4}, \mathrm{Al}_{6}, \mathrm{Al}_{8}, \mathrm{Al}_{9}, \mathrm{Al}_{11}\right.$ and $\left.\mathrm{Al}_{12}\right)$ colonies. On barley meal medium colonies colours of isolates were found dirty white ( $\left.\mathrm{Al}_{9}\right)$, light brown $\left(\mathrm{Al}_{1}, \mathrm{Al}_{2}, \mathrm{Al}_{4}, \mathrm{Al}_{6}, \mathrm{Al}_{7}, \mathrm{Al}_{8}\right.$, $\mathrm{Al}_{10}$, and $\left.\mathrm{Al}_{11}\right)$ and brown $\left(\mathrm{Al}_{3}, \mathrm{Al}_{5}, \mathrm{Al}_{7}\right.$ and $\left.\mathrm{Al}_{12}\right)$. Linseed leaf extract medium the colonies colours of 2 isolates $\left(\mathrm{Al}_{1}\right.$ and $\mathrm{Al}_{2}$ ) were recorded brown, $8\left(\mathrm{Al}_{3}, \mathrm{Al}_{4}, \mathrm{Al}_{5}, \mathrm{Al}_{6}, \mathrm{Al}_{7}, \mathrm{Al}_{9}, \mathrm{Al}_{10}\right.$ and $\left.\mathrm{Al}_{12}\right)$ light brown, remaining 2 whitish $\left(\mathrm{Al}_{11}\right)$ and dirty white $\left(\mathrm{Al}_{8}\right)$, respectively. On carrot agar medium variable colonies colours were also seen, in case of different isolates. 
Seven isolates $\left(\mathrm{Al}_{4}, \mathrm{Al}_{6}, \mathrm{Al}_{7}, \mathrm{Al}_{8}, \mathrm{Al}_{9}, \mathrm{Al}_{10}\right.$ and $\left.\mathrm{Al}_{11}\right)$ gave light brown, four $\left(\mathrm{Al}_{1}, \mathrm{Al}_{2}, \mathrm{Al}_{3}\right.$ and $\left.\mathrm{Al}_{5}\right)$ brown and one $\left(\mathrm{Al}_{12}\right)$ dirty white colour (Table.1\& Fig.1, 2,3,4,5 and 6). Sharma et al. (2013) studied the on cultural variability of different isolates of A. brassicae, collected from different part of country, by using potato dextrose medium, cauliflower (host) agar medium and carrot potato agar medium and reported variable colonies colour. Besides these, several worker working with different species of Alternaria, causing blight disease in different crop have also studied the variability of colony colour on different medium and reported variability in colour of colonies produced by different isolate time to time (Vishwanath, 1999, Kumar et al. 2007, Singh et al.2009, Khulbeet al. 2009, Meena et al. 2012, Pramila et al. 2014 and Singh et al. 2014). Variability in colonies colour, recorded in present studies supports the finding of earlier workers. The medium used for the studies in case of Alternaria lini is of new kind because; it has not been used earlier by other workers in their studies, in case of other Alternaria spp. causing disease in different crops.

Radial growth of colonies formed by different isolates of A. lini were recorded, after 7 days of inoculation and found variable on different media. On potato dextrose agar medium radial growth of colonies, ranged between $25 \mathrm{~mm}$ $\left(\mathrm{Al}_{12}\right)$ to $52 \mathrm{~mm}\left(\mathrm{Al}_{11}\right)$, on radish agar medium from $25 \mathrm{~mm}\left(\mathrm{Al}_{4}\right)$ to $46 \mathrm{~mm}\left(\mathrm{Al}_{10}\right)$, on maize meal medium from $25 \mathrm{~mm}$ $\left(\mathrm{Al}_{12}\right)$ to $50 \mathrm{~mm}\left(\mathrm{Al}_{5}\right)$, on barley meal medium from $30 \mathrm{~mm}\left(\mathrm{Al}_{12}\right)$ to $50 \mathrm{~mm}\left(\mathrm{Al}_{11}\right)$, on linseed leaf extract medium from 38 $\mathrm{mm}\left(\mathrm{Al}_{6}\right)$ to $60 \mathrm{~mm}\left(\mathrm{Al}_{11}\right)$ and on carrot agar medium from $34 \mathrm{~mm}\left(\mathrm{Al}_{12}\right)$ to $48 \mathrm{~mm}\left(\mathrm{Al}_{3}\right)$. The isolates $\mathrm{Al}_{11}$ collected from Berhampur (W. B.), showed maximum radial growth and isolate $\mathrm{Al}_{6}$ obtained from Kanpur (U.P.) showed minimum growth on all the test media (Table $2 \&$ Figure.1, 2,3,4,5 and 6). The shape of colonies formed by all the isolates on each media was noted circular. Variations in colonies growth (slow medium and fast) were also noted in different isolates on all the test media (Table.3\& Figure.1, 2,3,4,5 and 6). All the isolates except $\mathrm{Al}_{11}$ showed smooth margin of the colonies during their growth on potato dextrose agar medium, maize meal medium, linseed leaf extract medium, radish agar medium and carrot agar medium. Isolate $\mathrm{Al}_{11}$ also showed smooth margin on these media except radish agar medium and maize meal medium on which it showed rough margin. All the isolates showed wavy margin on barley meal medium. Colonies formed by different isolates also showed variable appearance (cottony, feathery, fluffy and compressed \& thin) during their growth. On maize meal medium all the isolates showed compressed \& thin appearance. It may be due less availability of nutrients or organic matter in this crop (Table.4\& Figure.1, 2,3,4,5 and 6). Most of the isolates under studies showed zonation on different media, only few have not shown zonation on some media during their colonies growth. Sporulation in different isolates on different media was also found variable. Some isolates showed early sporulation, some medium, some showed late sporulation on different media. On PDA most of the isolates, showed early sporulation except $\mathrm{Al}_{4}$ and $\mathrm{Al}_{6}$ which showed medium sporulation. On maize meal, medium and barley meal medium all the isolates showed medium sporulation. It is not as early as, in case of PDA. On radish agar medium all the isolates showed early sporulation except $\mathrm{Al}_{6}$ and $\mathrm{Al}_{10}$. Isolates on rest of the media showed either early or medium or late sporulation.

Concurrent with present findings, studies were also carried out by several workers in case of Alternariabrassicae isolates collected from different part of the country on variability of radial growth, colonies shape, colonies growth pattern, margin, appearance, zonation and sporulation and they reported $28.20 \mathrm{~mm}$ to $81.10 \mathrm{~mm}$ radial growth, circular to wavy shape, feathery, fluffy to cottony appearance of colonies, presence or absence of zonation, and early, medium to late sporulation on potato dextrose agar medium (Khulbeet al. 2009., Goyelet al.2011., Sharma et al.2013 and Pramila et al.2014). Kumar et al. (2009) and Singh et al. (2014) have also obtained the variability in case of Alternariasolani isolates and reported variation in radial growth (14.90 to 35.50), colour of colonies (brownish black, greenish black, redish black and yellowish), shape (circular to irregular), growth pattern (slow, medium and fast), margin (smooth, rough and wavy), 
appearance (fluffy, feathery and cottony), zonation (presence and absence) and sporulation (early, medium and late).

Table.1: Effect of Different Culture Media on Colony Colour of Different Isolates of Alternaria Lini

\begin{tabular}{|l|l|l|l|l|l|l|}
\hline $\begin{array}{c}\text { Media } \\
\text { Isolates }\end{array}$ & \multicolumn{1}{|c|}{ PDA } & $\begin{array}{c}\text { Radish } \\
\text { Agar } \\
\text { Medium }\end{array}$ & $\begin{array}{c}\text { Maize Meal } \\
\text { Medium }\end{array}$ & $\begin{array}{c}\text { Barley } \\
\text { Meal } \\
\text { Medium }\end{array}$ & $\begin{array}{c}\text { Linseed } \\
\text { Leaf } \\
\text { Extract } \\
\text { Medium }\end{array}$ & $\begin{array}{c}\text { Carrot } \\
\text { Agar } \\
\text { Medium }\end{array}$ \\
\hline $\mathrm{Al}_{1}$ & Brown & Brown & Dirty white & $\begin{array}{l}\text { Light } \\
\text { brown }\end{array}$ & Brown & Brown \\
\hline $\mathrm{Al}_{2}$ & Brown & Brown & Dirty white & $\begin{array}{l}\text { Light } \\
\text { brown }\end{array}$ & Brown & Brown \\
\hline $\mathrm{Al}_{3}$ & $\begin{array}{l}\text { Light } \\
\text { brown }\end{array}$ & Brown & Light brown & Brown & $\begin{array}{l}\text { Light } \\
\text { brown }\end{array}$ & Brown \\
\hline $\mathrm{Al}_{4}$ & Dirty white & $\begin{array}{l}\text { Light } \\
\text { brown }\end{array}$ & Light brown & $\begin{array}{l}\text { Light } \\
\text { brown }\end{array}$ & $\begin{array}{l}\text { Light } \\
\text { brown }\end{array}$ & Light brown \\
\hline $\mathrm{Al}_{5}$ & $\begin{array}{l}\text { Light } \\
\text { brown }\end{array}$ & Brown & Dirty white & Brown & $\begin{array}{l}\text { Light } \\
\text { brown }\end{array}$ & Brown \\
\hline $\mathrm{Al}_{6}$ & Dirty white & $\begin{array}{l}\text { Light } \\
\text { brown }\end{array}$ & Light brown & $\begin{array}{l}\text { Light } \\
\text { brown }\end{array}$ & $\begin{array}{l}\text { Light } \\
\text { brown }\end{array}$ & Light brown \\
\hline $\mathrm{Al}_{7}$ & $\begin{array}{l}\text { Light } \\
\text { brown }\end{array}$ & $\begin{array}{l}\text { Light } \\
\text { brown }\end{array}$ & Light brown & Brown & $\begin{array}{l}\text { Light } \\
\text { brown }\end{array}$ & Light brown \\
\hline $\mathrm{Al}_{8}$ & Whitish & $\begin{array}{l}\text { Light } \\
\text { brown }\end{array}$ & Light brown & $\begin{array}{l}\text { Light } \\
\text { brown }\end{array}$ & $\begin{array}{l}\text { Dirty } \\
\text { white }\end{array}$ & Light brown \\
\hline $\mathrm{Al}_{9}$ & Dirty white & $\begin{array}{l}\text { Light } \\
\text { brown }\end{array}$ & Light brown & $\begin{array}{l}\text { Dirty } \\
\text { hhite }\end{array}$ & $\begin{array}{l}\text { Light } \\
\text { brown }\end{array}$ & Light brown \\
\hline $\mathrm{Al}_{10}$ & Dirty white & $\begin{array}{l}\text { Dirty } \\
\text { white }\end{array}$ & Whitish & $\begin{array}{l}\text { Light } \\
\text { brown }\end{array}$ & $\begin{array}{l}\text { Light } \\
\text { brown }\end{array}$ & Light brown \\
\hline $\mathrm{Al}_{11}$ & $\begin{array}{l}\text { Light } \\
\text { brown }\end{array}$ & $\begin{array}{l}\text { Light } \\
\text { brown }\end{array}$ & Light brown & $\begin{array}{l}\text { Light } \\
\text { brown }\end{array}$ & Whitish & Light brown \\
\hline $\mathrm{Al}_{12}$ & $\begin{array}{l}\text { Light } \\
\text { brown }\end{array}$ & $\begin{array}{l}\text { Light } \\
\text { brown }\end{array}$ & Light brown & Brown & $\begin{array}{l}\text { Light } \\
\text { brown }\end{array}$ & Dirty white \\
\hline
\end{tabular}

Table 2: Effect of Different Culture Media on the Mycelial Growth of Different Isolates of Alternaria Lini (Mm.)

\begin{tabular}{|l|l|l|l|l|l|l|}
\hline $\begin{array}{c}\text { Media } \\
\text { Isolates }\end{array}$ & PDA & $\begin{array}{c}\text { Radish } \\
\text { Agar } \\
\text { Medium }\end{array}$ & $\begin{array}{c}\text { Maize } \\
\text { Meal } \\
\text { Medium }\end{array}$ & $\begin{array}{c}\text { Barley } \\
\text { Meal } \\
\text { Medium }\end{array}$ & $\begin{array}{c}\text { Linseed } \\
\text { Leaf } \\
\text { Extract } \\
\text { Medium }\end{array}$ & $\begin{array}{c}\text { Carrot } \\
\text { Agar } \\
\text { Medium }\end{array}$ \\
\hline $\mathbf{A l}_{\mathbf{1}}$ & 45 & 44 & 45 & 40 & 53 & 45 \\
\hline $\mathbf{A l}_{\mathbf{2}}$ & 45 & 44 & 45 & 42 & 52 & 44 \\
\hline $\mathbf{A l}_{\mathbf{3}}$ & 45 & 45 & 50 & 47 & 55 & 48 \\
\hline $\mathbf{A l}_{\mathbf{4}}$ & 35 & 25 & 28 & 30 & 40 & 34 \\
\hline $\mathbf{A l}_{\mathbf{5}}$ & 45 & 45 & 50 & 48 & 54 & 47 \\
\hline $\mathbf{A l}_{\mathbf{6}}$ & 34 & 26 & 28 & 30 & 38 & 34 \\
\hline $\mathbf{A l}_{\mathbf{7}}$ & 45 & 46 & 45 & 40 & 42 & 44 \\
\hline $\mathbf{A l}_{\mathbf{8}}$ & 44 & 45 & 44 & 42 & 40 & 42 \\
\hline $\mathbf{A l}_{\mathbf{9}}$ & 45 & 44 & 46 & 42 & 42 & 44 \\
\hline $\mathbf{A l}_{\mathbf{1 0}}$ & 46 & 46 & 45 & 40 & 40 & 40 \\
\hline $\mathbf{A l}_{\mathbf{1 1}}$ & 52 & 40 & 45 & 50 & 60 & 40 \\
\hline
\end{tabular}




\begin{tabular}{|l|l|l|l|l|l|l|}
\hline \multicolumn{7}{|c|}{ Table 2: Contd., } \\
\hline $\mathbf{A l}_{\mathbf{1 2}}$ & 25 & 28 & 25 & 30 & 45 & 34 \\
\hline S.Em \pm & 0.82 & 0.82 & 0.88 & 0.82 & 0.86 & 0.82 \\
\hline $\begin{array}{l}\text { C.D. at } \\
\mathbf{1 \%}\end{array}$ & 2.28 & 2.28 & 2.47 & 2.28 & 2.44 & 2.28 \\
\hline
\end{tabular}

Table .3 Variations in Colonies Growth of Different Isolates of Alternaria Lini on Different Culture Media

\begin{tabular}{|l|l|l|l|l|l|l|}
\hline $\begin{array}{c}\text { Media } \\
\text { Isolates }\end{array}$ & PDA & $\begin{array}{c}\text { Radish } \\
\text { Agar } \\
\text { Medium }\end{array}$ & $\begin{array}{c}\text { Maize } \\
\text { Meal } \\
\text { Medium }\end{array}$ & $\begin{array}{c}\text { Barley } \\
\text { Meal } \\
\text { Medium }\end{array}$ & $\begin{array}{c}\text { Linseed } \\
\text { Leaf } \\
\text { Extract } \\
\text { Medium }\end{array}$ & $\begin{array}{c}\text { Carrot } \\
\text { Agar } \\
\text { Medium }\end{array}$ \\
\hline $\mathrm{Al}_{1}$ & Medium & Medium & Medium & Medium & Fast & Medium \\
\hline $\mathrm{Al}_{2}$ & Medium & Medium & Medium & Medium & Fast & Medium \\
\hline $\mathrm{Al}_{3}$ & Medium & Medium & Fast & Medium & Fast & Medium \\
\hline $\mathrm{Al}_{4}$ & Slow & Slow & Slow & Slow & Medium & Slow \\
\hline $\mathrm{Al}_{5}$ & Medium & Medium & Fast & Medium & Fast & Medium \\
\hline $\mathrm{Al}_{6}$ & Slow & Slow & Slow & Slow & Medium & Slow \\
\hline $\mathrm{Al}_{7}$ & Medium & Slow & Medium & Medium & Medium & Medium \\
\hline $\mathrm{Al}_{8}$ & Medium & Medium & Medium & Medium & Medium & Medium \\
\hline $\mathrm{Al}_{9}$ & Medium & Medium & Medium & Medium & Medium & Medium \\
\hline $\mathrm{Al}_{10}$ & Medium & Medium & Medium & Medium & Medium & Medium \\
\hline $\mathrm{Al}_{11}$ & Fast & Medium & Medium & Fast & fast & Medium \\
\hline $\mathrm{Al}_{12}$ & Slow & Slow & Slow & Slow & Medium & Slow \\
\hline
\end{tabular}

Table.4: Effect of Different Culture Media on the Appearance of Colonies of Alternaria Lini Isolates

\begin{tabular}{|l|l|l|l|l|l|l|}
\hline $\begin{array}{c}\text { Media } \\
\text { Isolates }\end{array}$ & \multicolumn{1}{|c|}{ PDA } & $\begin{array}{c}\text { Radish } \\
\text { Agar } \\
\text { Medium }\end{array}$ & $\begin{array}{c}\text { Maize Meal } \\
\text { Medium }\end{array}$ & $\begin{array}{c}\text { Barley } \\
\text { Meal } \\
\text { Medium }\end{array}$ & $\begin{array}{c}\text { Linseed Leaf } \\
\text { Extract } \\
\text { Medium }\end{array}$ & $\begin{array}{c}\text { Carrot } \\
\text { Agar } \\
\text { Medium }\end{array}$ \\
\hline $\mathrm{AI}_{1}$ & $\begin{array}{l}\text { Thick } \\
\text { cottony }\end{array}$ & Cottony & $\begin{array}{l}\text { Compressed } \\
\text { and thin }\end{array}$ & Cottony & Cottony & Cottony \\
\hline $\mathrm{Al}_{2}$ & $\begin{array}{l}\text { Thick } \\
\text { cottony }\end{array}$ & Cottony & $\begin{array}{l}\text { Compressed } \\
\text { and thin }\end{array}$ & Cottony & Cottony & Cottony \\
\hline $\mathrm{Al}_{3}$ & Cottony & Cottony & $\begin{array}{l}\text { Compressed } \\
\text { and thin }\end{array}$ & Cottony & Cottony & Feathery \\
\hline $\mathrm{Al}_{4}$ & Feathery & Cottony & $\begin{array}{l}\text { Compressed } \\
\text { and thin }\end{array}$ & Cottony & Cottony & Cottony \\
\hline $\mathrm{Al}_{5}$ & Cottony & Cottony & $\begin{array}{l}\text { Compressed } \\
\text { and thin }\end{array}$ & Feathery & Cottony & Feathery \\
\hline $\mathrm{Al}_{6}$ & Cottony & Cottony & $\begin{array}{l}\text { Compressed } \\
\text { and thin }\end{array}$ & Cottony & Cottony & Cottony \\
\hline $\mathrm{Al}_{7}$ & Cottony & Cottony & $\begin{array}{l}\text { Compressed } \\
\text { and thin }\end{array}$ & Fluffy & Cottony & Feathery \\
\hline $\mathrm{Al}_{8}$ & Cottony & Feathery & $\begin{array}{l}\text { Compressed } \\
\text { and thin }\end{array}$ & Cottony & Cottony & Feathery \\
\hline $\mathrm{Al}_{9}$ & Cottony & Cottony & $\begin{array}{l}\text { Compressed } \\
\text { and thin }\end{array}$ & Cottony & Cottony & Feathery \\
\hline & & & & \\
\hline
\end{tabular}




\begin{tabular}{|l|l|l|l|l|l|l|}
\hline \multicolumn{7}{|c|}{ Table 4: Contd., } \\
\hline $\mathrm{Al}_{10}$ & Feathery & Cottony & $\begin{array}{l}\text { Compressed } \\
\text { and thin }\end{array}$ & Cottony & Cottony & Feathery \\
\hline $\mathrm{Al}_{11}$ & Cottony & Cottony & $\begin{array}{l}\text { Compressed } \\
\text { and thin }\end{array}$ & Fluffy & Cottony & Cottony \\
\hline $\mathrm{Al}_{12}$ & Cottony & Cottony & $\begin{array}{l}\text { Compressed } \\
\text { and thin }\end{array}$ & Cottony & Cottony & Cottony \\
\hline
\end{tabular}

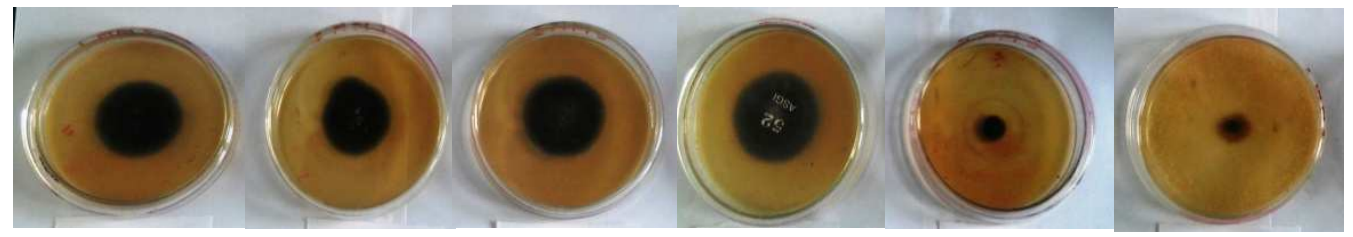
$\mathbf{A l}_{1}$
$\mathrm{AI}_{2}$
$\mathrm{AI}_{3}$
$\mathrm{AI}_{4}$
$\mathbf{A I}_{5}$
$\mathrm{AI}_{6}$
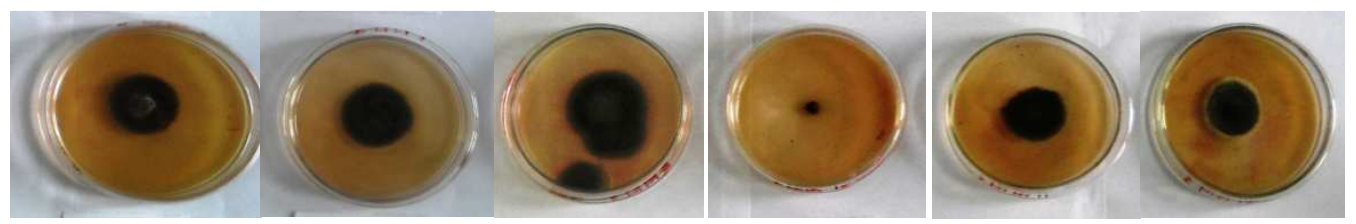

$\mathbf{A I}_{7}$

$\mathbf{A I}_{8}$

$\mathbf{A I}_{9}$

$\mathbf{A I}_{10}$

$\mathbf{A I}_{11}$

$\mathbf{A I}_{12}$

Figure 1: Colony Morphology of $\mathrm{A}$. Lini Isolates on Carrot Agar Medium $\left(\mathrm{Al}_{1} . \mathrm{Patna}, \mathrm{Al}_{2} \cdot \mathbf{B i l a s p u r ,}\right.$ $\mathrm{Al}_{3}$.Sultanpur, $\mathrm{Al}_{4}$.Mauranipur, $\mathrm{Al}_{5}$.Mashodha, $\mathrm{Al}_{6} \cdot \mathrm{Kanpur}, \mathrm{Al}_{7} \cdot \mathrm{Kangra}, \mathrm{Al}_{8}$.Ranchi, Al 9 .Mirzapur, $\mathrm{Al}_{10}$.Gorkhpur, $\mathrm{Al}_{11}$.Berhampur, $\mathrm{Al}_{12} \cdot$ Kumarganj)

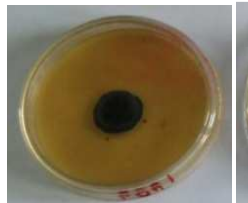

$\mathbf{A I}_{1}$

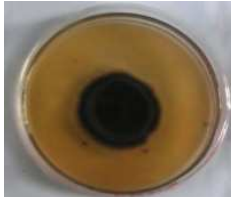

$\mathrm{AI}_{7}$

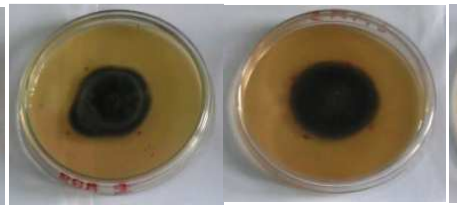

$\mathbf{A I}_{2}$

$\mathrm{AI}_{3}$

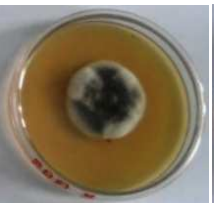

$\mathbf{A I}_{8}$

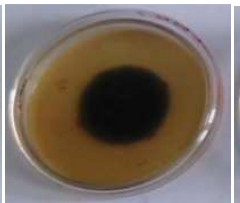

AI $\mathbf{I}_{9}$

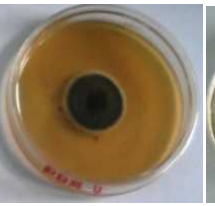

$\mathbf{A I}_{4}$

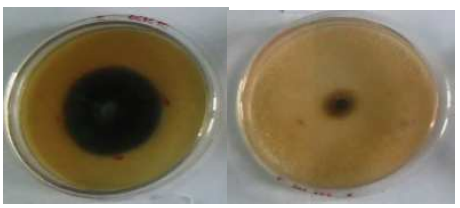

$\mathbf{A I}_{5}$

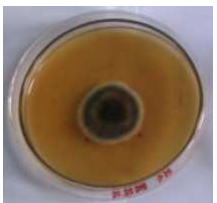

$\mathbf{A I}_{10}$

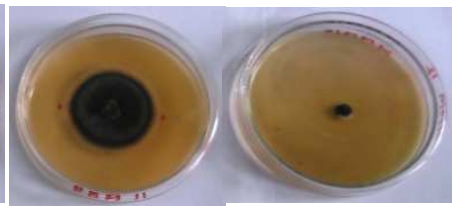

$\mathbf{A I}_{11}$

$\mathbf{A I}_{12}$

Figure.2: Colony Morphology of $\mathrm{A}$. Lini Isolates on Potato Dextrose Agar Medium ( $\mathrm{Al}_{1}$. Patna, $\mathrm{Al}_{2} . \mathrm{Bilaspur}$ $\mathrm{Al}_{3}$.Sultanpur, $\mathrm{Al}_{4}$.Mauranipur, $\mathrm{Al}_{5}$.Mashodha, $\mathrm{Al}_{6} \cdot$ Kanpur, $\mathrm{Al}_{7}$.Kangra, $\mathrm{Al}_{8}$.Ranchi, $A l_{9}$.Mirzapur, $\mathbf{A l}_{10}$.Gorkhpur, $\mathbf{A l}_{11}$.Berhampur, $\mathbf{A l}_{12}$. Kumarganj)

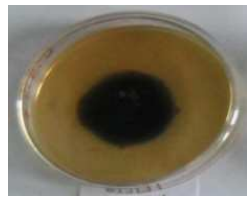

$\mathrm{AI}_{1}$

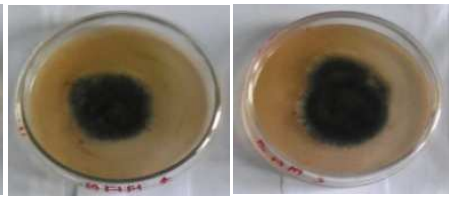

$\mathbf{A I}_{2}$
$\mathrm{AI}_{3}$

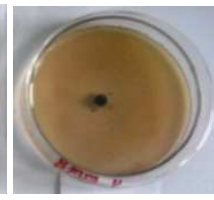

$\mathrm{AI}_{4}$

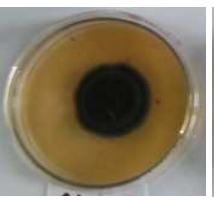

$\mathbf{A I}_{5}$

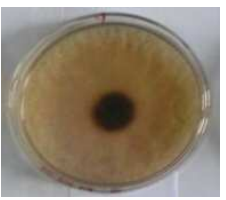

$\mathrm{AI}_{6}$ 


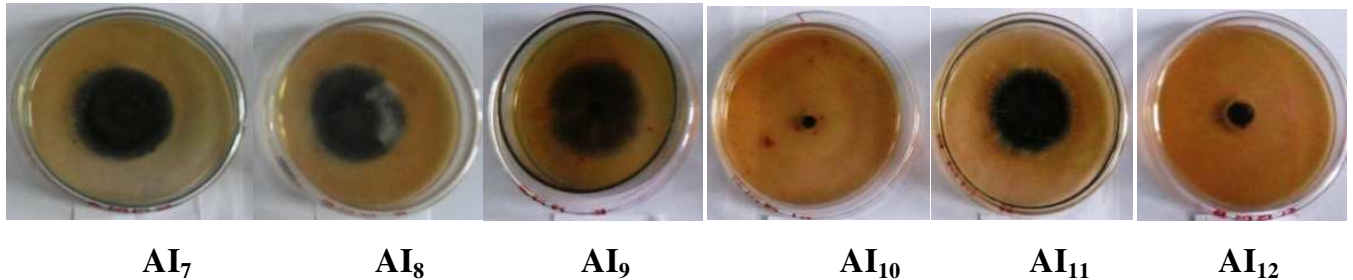

Figure 3: Colony morphology of $A$. lini isolates on Barley meal medium $\left(\mathrm{Al}_{1} . \mathrm{Patna}_{\mathbf{2}} \mathrm{Al}_{2} \cdot \mathrm{Bilaspur}, \mathrm{Al}_{3} \cdot \mathrm{Sultanpur}\right.$, $\mathrm{Al}_{4} \cdot$ Mauranipur, $\mathrm{Al}_{5} \cdot \mathrm{Mashodha}, \mathrm{Al}_{6} \cdot \mathrm{Kanpur}, \mathrm{Al}_{7} \cdot \mathrm{Kangra}, \mathrm{Al}_{8} \cdot \mathrm{Ranchi}$,

$\mathrm{Al}_{9}$.Mirzapur, $\mathrm{Al}_{10}$.Gorkhpur, $\mathrm{Al}_{11}$.Berhampur, $\mathrm{Al}_{12}$. Kumarganj)
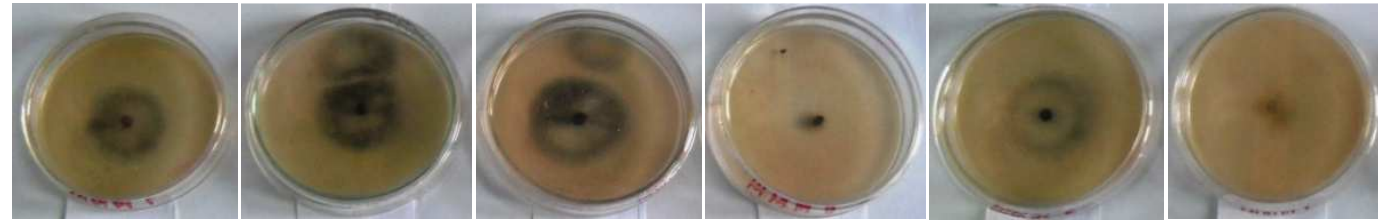

$\mathrm{AI}_{1}$

$\mathrm{AI}_{2}$

$\mathbf{A I}_{3}$

$\mathrm{AI}_{4}$

$\mathrm{AI}_{5}$

$\mathrm{AI}_{6}$
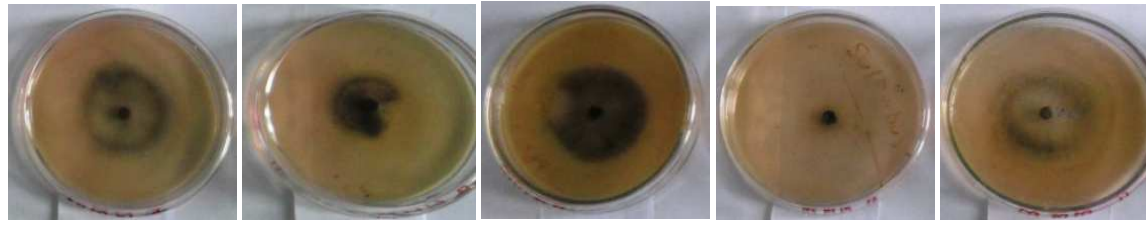

$\mathbf{A I}_{7}$

$\mathbf{A I}_{8}$

AI

A $\mathbf{I}_{10}$

$\mathbf{A I}_{11}$

$\mathbf{A I}_{12}$

Figure 4: Colony Morphology of $\boldsymbol{A}$. Lini Isolates on Maize Meal Medium ( $\mathrm{Al}_{1}$.Patna, $\mathbf{A l}_{2} \cdot \mathbf{B i l a s p u r}$, $\mathrm{Al}_{3}$. Sultanpur, $\mathrm{Al}_{4} \cdot$ Mauranipur, $\mathrm{Al}_{5} \cdot \mathrm{Mashodha,} \mathrm{Al}_{6} \cdot \mathrm{Kanpur}, \mathrm{Al}_{7} \cdot \mathrm{Kangra}, \mathrm{Al}_{\mathbf{8}} \cdot \mathrm{Ranchi}_{\text {, }}$ $\mathrm{Al}_{9}$.Mirzapur, $\mathrm{Al}_{10}$.Gorkhpur, $\mathrm{Al}_{11}$.Berhampur, $\mathrm{Al}_{12}$. Kumarganj)

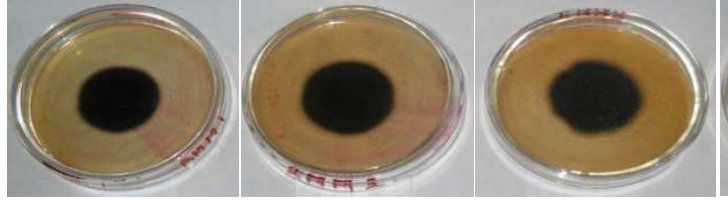

$\mathbf{A I}_{1}$

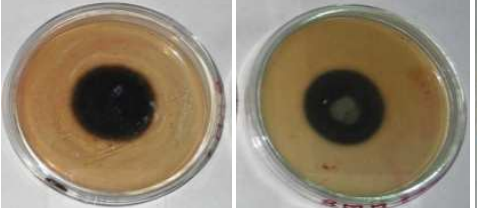

$\mathbf{A I}_{7}$

$\mathbf{A I}_{8}$

$\mathbf{A I}_{3}$

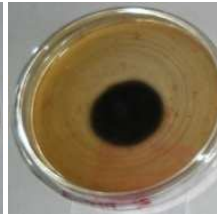

AI 9

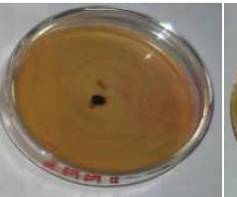

$\mathrm{AI}_{4}$

$\mathbf{A I}_{5}$

$\mathbf{A I}_{6}$

Figure 5: Colony morphology of $A$. lini isolates on Radish agar medium $\left(\mathrm{Al}_{1} . \mathbf{P a t n a}_{\mathbf{2}} \mathbf{A l}_{2} \cdot \mathbf{B i l a s p u r}\right.$, $\mathrm{Al}_{3}$.Sultanpur, $\mathrm{Al}_{4} \cdot$ Mauranipur, $\mathrm{Al}_{5} \cdot \mathrm{Mashodha,} \mathrm{Al}_{6} \cdot$ Kanpur, $\mathrm{Al}_{7} \cdot \mathrm{Kangra}, \mathrm{Al}_{8} \cdot \mathrm{Ranchi}$ Al 9 .Mirzapur, $\mathrm{Al}_{10}$.Gorkhpur, $\mathrm{Al}_{11}$.Berhampur, $\mathrm{Al}_{12}$. Kumarganj)

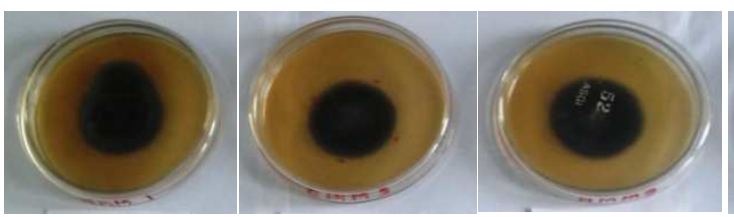

$\mathbf{A I}_{1}$
$\mathbf{A I}_{2}$

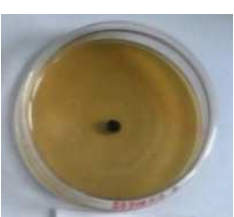

$\mathbf{A I}_{4}$

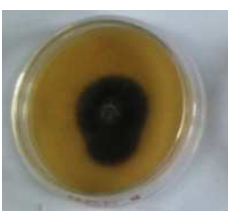

$\mathbf{A I}_{5}$

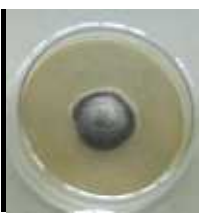

$\mathbf{A I}_{6}$ 


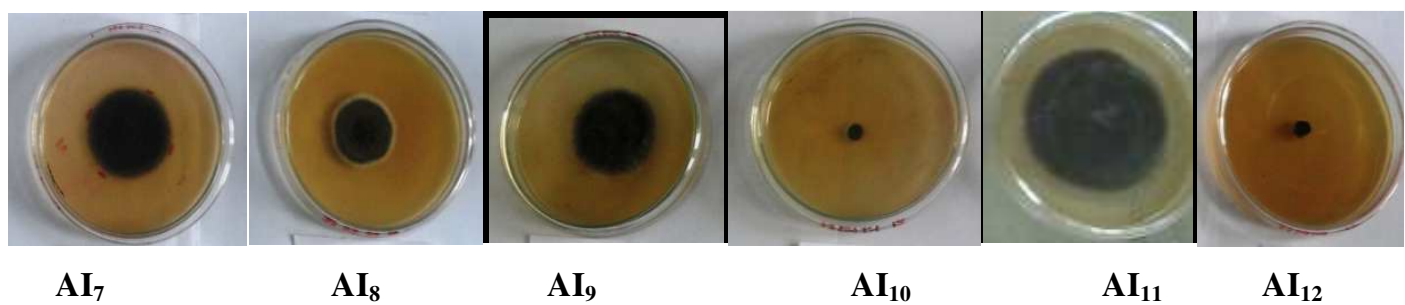

Figure 6: Colony Morphology of $\mathrm{A}$. Lini Isolates on Linseed Leaf Extract Medium $\left(\mathrm{Al}_{1}\right.$. Patna, $\mathrm{Al}_{2} . \mathrm{Bilaspur}$, $\mathrm{Al}_{3}$. Sultanpur, $\mathrm{Al}_{4} \cdot \mathrm{Mauranipur,} \mathrm{Al}_{5} \cdot \mathrm{Mashodha,} \mathrm{Al}_{6} \cdot \mathrm{Kanpur}, \mathrm{Al}_{7} \cdot \mathrm{Kangra}, \mathrm{Al}_{8} \cdot \mathbf{R a n c h i}$, $\mathrm{Al}_{9}$.Mirzapur, $\mathbf{A l}_{10}$.Gorkhpur, $\mathrm{Al}_{11}$.Berhampur, $\mathrm{Al}_{12}$. Kumarganj)

\section{Morphological variability}

Besides cultural variability of isolates on different culture media, morphological variability of conidia and conidiophores produced by them were also studied and discussed. Average length of conidia of Alternarialini, obtained from different isolates ranged between $23.26 \mu \mathrm{m}\left(\mathrm{Al}_{7}\right.$ of Kangra) to $45.72 \mu \mathrm{m}\left(\mathrm{Al}_{11}\right.$ of Berhampur) and width between 6.76 $\mu \mathrm{m}\left(\mathrm{Al}_{4}\right.$ of Mauranipur) to $17.01 \mu \mathrm{m}$ ( $\mathrm{Al}_{11}$ of Berhampur). Horizontal septa in conidia, obtained from different isolates were also found variable, which ranged between, on basis of mean from $1.86\left(\mathrm{Al}_{8}\right.$ of Ranchi) to $5.60\left(\mathrm{Al}_{11}\right.$ of $\left.\mathrm{Berhampur}\right)$. Likewise, colonies colour and shape of conidia, also found variable in different isolates. Most of the conidia were found light brown colour $\left(\mathrm{Al}_{1}, \mathrm{Al}_{2}, \mathrm{Al}_{3}, \mathrm{Al}_{4}, \mathrm{Al}_{6}, \mathrm{Al}_{9}, \mathrm{Al}_{11}\right.$ and $\left.\mathrm{Al}_{12}\right)$ and few were dark brown in colour $\left(\mathrm{Al}_{5}, \mathrm{Al}_{7}, \mathrm{Al}_{8}\right.$, and $\left.\mathrm{Al}_{10}\right)$. Oval shape of conidia were observed in isolates $\mathrm{Al}_{1}, \mathrm{Al}_{2}, \mathrm{Al}_{4}, \mathrm{Al}_{5}, \mathrm{Al}_{6}, \mathrm{Al}_{7}$ and $\mathrm{Al}_{10}$ obclavate type in isolates $\mathrm{Al}_{3}, \mathrm{Al}_{8}$ and $\mathrm{Al}_{9}$, while $\mathrm{Al}_{11}$ and $\mathrm{Al}_{12}$ were noted clavate type. In general, the conidia obtained from most of the isolates on PDA were unbeaked, some were with rudimentary beaked and few were short beaked. Unbeaked conidia were obtained from isolates $\mathrm{Al}_{1}, \mathrm{Al}_{2}, \mathrm{Al}_{4}, \mathrm{Al}_{5}, \mathrm{Al}_{6}, \mathrm{Al}_{7}, \mathrm{Al}_{8}$, and $\mathrm{Al}_{11}$, short beaked from $\mathrm{Al}_{3}$ and $\mathrm{Al}_{9}$, while rudimentary beaked from $\mathrm{Al}_{11}$ and $\mathrm{Al}_{12}$,respectively(Table.5 and Fig.7). Concurrent with present finding Kumar et al. (2003) have also reported distinct appearance among the isolates of Alternaria brassicae of rapeseed- mustard, in terms of conidial length, width and number of septation at Haryana. They reported that average conidial length varied appreciably from $118.20 \mu \mathrm{m}$ to $194.52 \mu \mathrm{m}$ and identified 8 pathotypes. Mehta et al. (2003) worked on the morphological and pathological variation in rapeseed- mustard isolates of $A$. brassicae collected from different agroclimatic zone of India. They also reported variations in spore length and width, and indicated the existence of variability in the pathogen. Khan et al. (2007) isolated A. brassicae from the samples collected from different places of Aligarh district and reported variations in conidial length (112 to $185.60 \mu \mathrm{m})$, width $(14.40 \mu \mathrm{m}$ to $17.60 \mu \mathrm{m})$ and septation (5 to 16). Singh et al. (2009) also studied on morphological variation among 105 isolates A. brassicae collected from 18 district of Haryana. They found that, average conidial length and width ranged from 117.00 to $192 \mu \mathrm{m}$ and 14.0 to $24 \mu \mathrm{m}$, respectively. Length of beak, horizontal septa and vertical septa varied from 42.0 to $116.0 \mu \mathrm{m}, 6$ to 9 and 1 to 3, respectively. Goyalet al. (2011) and Sharma et al. (2013), also found variable morphology (length, width and number of septa) in different isolates of A. brassicae collected from different parts of country. Khulbe et al. (2011) and Pramila et al. (2014), studied the variability in different isolates of A. brassicae, collected from different part of Uttarakhand. They reported that conidial size ranged from 55.23 to $152.17 \mu \mathrm{m} \times 12.00$ to $88.40 \mu \mathrm{m}$ and 105 to $135 \times 10$ to $20 \mu \mathrm{m}$, respectively. The variation in conidial length, width, septa and beak, in case of $A$. lini in present studies supports the views of earlier workers. The studies therefore, indicate the existence of variability among the A. lini isolates collected from different part of country. 
Like the conidial characters of A. lini morphological variability in conidiophores, were also recorded in respect of length, width, colour and number of septa. Average length and width of conidiophores, ranged between $33.00 \mu \mathrm{m}\left(\mathrm{Al}_{7}\right)$ to $81.50 \mu \mathrm{m}\left(\mathrm{Al}_{12}\right)$ and $4.45 \mu \mathrm{m}\left(\mathrm{Al}_{7}\right)$ to $9.27 \mu \mathrm{m}\left(\mathrm{Al}_{3}\right)$, respectively. Colour of conidiophores was observed light brown to dark brown. Average septation in conidiophores, ranged between 3.33 ( $\mathrm{Al}_{7}$ of Kangra) to $7.53\left(\mathrm{Al}_{12}\right.$ of Kumarganj) (Table.6).

As per literature available, it was noted that most of the earlier pathologist working with different Alternariaspp., have studied the variations in conidial characters only. None have taken into consideration, about the variation in characters of conidiophores. During present studies, variation in characters of conidiophores have also been taken into consideration and found variable.

Table 5: Morphological Characters of Conidia

\begin{tabular}{|c|c|c|c|c|c|c|c|c|c|}
\hline \multirow{2}{*}{$\begin{array}{c}\text { Different } \\
\text { isolates }\end{array}$} & \multicolumn{2}{|c|}{ Length $(\mu \mathrm{m})$} & \multicolumn{2}{|c|}{ Width $(\mu \mathrm{m})$} & \multirow{2}{*}{ Colour } & \multirow{2}{*}{ Shape } & \multicolumn{2}{|c|}{ Horizontal septa } & \multirow{2}{*}{ Beak } \\
\hline & Range & $\begin{array}{l}\text { Ave } \\
\text { rage }\end{array}$ & Range & $\begin{array}{l}\text { Ave } \\
\text { rage }\end{array}$ & & & $\begin{array}{l}\text { Rang } \\
\text { e }\end{array}$ & Average & \\
\hline $\mathrm{Al}_{1}$ & $\begin{array}{l}22.50- \\
30.00\end{array}$ & $\begin{array}{c}27.3 \\
3 \\
\end{array}$ & $\begin{array}{c}14.00- \\
15.50 \\
\end{array}$ & $\begin{array}{c}15.0 \\
0 \\
\end{array}$ & $\begin{array}{l}\text { Light } \\
\text { brown }\end{array}$ & oval & $2-3$ & 2.60 & Unbeaked \\
\hline $\mathrm{Al}_{2}$ & $\begin{array}{l}22.50- \\
26.50 \\
\end{array}$ & $\begin{array}{c}23.7 \\
3 \\
\end{array}$ & $\begin{array}{l}11.30- \\
15.00 \\
\end{array}$ & $\begin{array}{c}14.0 \\
1 \\
\end{array}$ & $\begin{array}{l}\text { Light } \\
\text { brown }\end{array}$ & oval & $3-4$ & 3.13 & Unbeaked \\
\hline $\mathrm{Al}_{3}$ & $\begin{array}{l}22.50- \\
30.00\end{array}$ & $\begin{array}{c}28.0 \\
0\end{array}$ & $\begin{array}{l}7.50- \\
11.33 \\
\end{array}$ & 9.02 & $\begin{array}{l}\text { Dark } \\
\text { brown }\end{array}$ & $\begin{array}{l}\text { obclav } \\
\text { ate }\end{array}$ & $2-3$ & 2.40 & $\begin{array}{l}\begin{array}{l}\text { Short } \\
\text { beaked }\end{array} \\
\end{array}$ \\
\hline $\mathrm{Al}_{4}$ & $\begin{array}{l}30.00- \\
33.80\end{array}$ & $\begin{array}{c}30.7 \\
6 \\
\end{array}$ & $3.80-7.50$ & 6.76 & $\begin{array}{l}\text { Light } \\
\text { brown }\end{array}$ & oval & $2-3$ & 2.33 & Unbeaked \\
\hline $\mathrm{Al}_{5}$ & $\begin{array}{l}22.50- \\
30.00\end{array}$ & $\begin{array}{c}26.5 \\
0\end{array}$ & $\begin{array}{l}7.50- \\
11.30 \\
\end{array}$ & 8.70 & $\begin{array}{l}\text { Dark } \\
\text { brown }\end{array}$ & oval & $3-4$ & 3.40 & Unbeaked \\
\hline $\mathrm{Al}_{6}$ & $\begin{array}{l}22.50- \\
30.00\end{array}$ & $\begin{array}{c}28.0 \\
0\end{array}$ & $\begin{array}{l}7.50- \\
11.30 \\
\end{array}$ & 8.26 & $\begin{array}{l}\text { Light } \\
\text { brown }\end{array}$ & oval & $2-3$ & 2.46 & Unbeaked \\
\hline $\mathrm{Al}_{7}$ & $\begin{array}{l}22.50- \\
26.30\end{array}$ & $\begin{array}{c}23.2 \\
6\end{array}$ & $\begin{array}{l}15.00- \\
18.80\end{array}$ & $\begin{array}{c}15.7 \\
6\end{array}$ & $\begin{array}{l}\text { Dark } \\
\text { brown }\end{array}$ & oval & $2-3$ & 2.86 & Unbeaked \\
\hline $\mathrm{Al}_{8}$ & $\begin{array}{l}30.00- \\
33.80\end{array}$ & $\begin{array}{c}31.0 \\
1\end{array}$ & $7.0-8.0$ & 7.50 & $\begin{array}{l}\text { Dark } \\
\text { brown }\end{array}$ & $\begin{array}{l}\text { obclav } \\
\text { ate }\end{array}$ & $1-2$ & 1.86 & Unbeaked \\
\hline $\mathrm{Al}_{9}$ & $\begin{array}{l}22.50- \\
26.30\end{array}$ & $\begin{array}{c}24.0 \\
4\end{array}$ & $\begin{array}{c}15.00- \\
18.80 \\
\end{array}$ & $\begin{array}{c}16.0 \\
6 \\
\end{array}$ & $\begin{array}{l}\text { Light } \\
\text { brown }\end{array}$ & $\begin{array}{l}\text { obclav } \\
\text { ate }\end{array}$ & $3-4$ & 3.53 & $\begin{array}{l}\text { Short } \\
\text { beaked }\end{array}$ \\
\hline $\mathrm{Al}_{10}$ & $\begin{array}{l}22.50- \\
30.00\end{array}$ & $\begin{array}{c}27.0 \\
0 \\
\end{array}$ & $\begin{array}{l}7.50- \\
11.30 \\
\end{array}$ & 8.00 & $\begin{array}{l}\text { Dark } \\
\text { brown }\end{array}$ & oval & $2-3$ & 2.46 & Unbeaked \\
\hline $\mathrm{Al}_{11}$ & $\begin{array}{c}45.00- \\
48.00\end{array}$ & $\begin{array}{c}45.7 \\
2 \\
\end{array}$ & $\begin{array}{c}15.00- \\
18.80 \\
\end{array}$ & $\begin{array}{c}17.0 \\
1 \\
\end{array}$ & $\begin{array}{l}\text { Light } \\
\text { brown }\end{array}$ & clavate & $5-7$ & 5.60 & $\begin{array}{l}\text { Rudimentar } \\
\text { y beaked }\end{array}$ \\
\hline $\mathrm{Al}_{12}$ & $\begin{array}{l}22.50- \\
30.00\end{array}$ & $\begin{array}{c}25.5 \\
0\end{array}$ & $\begin{array}{l}7.50- \\
11.30 \\
\end{array}$ & $\begin{array}{c}10.0 \\
0\end{array}$ & $\begin{array}{l}\text { Dark } \\
\text { brown }\end{array}$ & clavate & $2-3$ & 2.52 & $\begin{array}{l}\text { Rudimento } \\
\text { ry beaked }\end{array}$ \\
\hline S.Em \pm & & 0.62 & & 0.30 & & & & 0.17 & \\
\hline $\begin{array}{l}\text { C.D.at } \\
1 \%\end{array}$ & & 1.74 & & 0.83 & & & & 0.48 & \\
\hline
\end{tabular}

Table 6: Morphological Characters of Conidiophores

\begin{tabular}{|l|c|c|c|c|c|c|c|}
\hline \multirow{2}{*}{$\begin{array}{c}\text { Different } \\
\text { isolates }\end{array}$} & \multicolumn{2}{|c|}{ Length $(\mu \mathrm{m})$} & \multicolumn{2}{|c|}{ Width $(\boldsymbol{\mu m})$} & \multicolumn{2}{|c|}{ Colour } & \multicolumn{2}{c|}{ Horizontal septa } \\
\cline { 2 - 5 } \cline { 3 - 4 } & Range & $\begin{array}{c}\text { Ave } \\
\text { rage }\end{array}$ & Range & Average & & Range & Average \\
\hline $\mathrm{Al}_{1}$ & $60.00-67.50$ & $\begin{array}{c}63.5 \\
0\end{array}$ & $3.80-7.50$ & 5.52 & Dark brown & $4-5$ & 4.26 \\
\hline $\mathrm{Al}_{2}$ & $60.00-67.50$ & $\begin{array}{c}62.5 \\
0\end{array}$ & $3.80-7.50$ & 5.77 & Dark brown & $4-5$ & 4.26 \\
\hline
\end{tabular}




\begin{tabular}{|c|c|c|c|c|c|c|c|}
\hline \multicolumn{8}{|c|}{ Table 6: Contd., } \\
\hline $\mathrm{Al}_{3}$ & $75.00-90.00$ & $\begin{array}{c}79.5 \\
0\end{array}$ & $\begin{array}{l}7.50- \\
11.30\end{array}$ & 9.27 & Dark brown & $6-8$ & 6.83 \\
\hline $\mathrm{Al}_{4}$ & $52.50-60.00$ & $\begin{array}{c}56.5 \\
0\end{array}$ & $6.00-7.50$ & 7.10 & Light brown & $5-7$ & 6.09 \\
\hline $\mathrm{Al}_{5}$ & $75.00-82.90$ & $\begin{array}{c}78.0 \\
0 \\
\end{array}$ & $\begin{array}{l}7.50- \\
11.30 \\
\end{array}$ & 9.02 & Dark brown & $6-8$ & 6.86 \\
\hline $\mathrm{Al}_{6}$ & $45.00-48.80$ & $\begin{array}{c}46.2 \\
6 \\
\end{array}$ & $6.00-7.50$ & 7.10 & Light Brown & $3-4$ & 3.53 \\
\hline $\mathrm{Al}_{7}$ & $30.00-37.50$ & $\begin{array}{c}33.0 \\
0\end{array}$ & $3.80-7.50$ & 4.54 & Light brown & $3-4$ & 3.33 \\
\hline $\mathrm{Al}_{8}$ & $33.80-37.50$ & $\begin{array}{c}36.0 \\
0\end{array}$ & $6.00-7.50$ & 7.00 & Dark brown & $3-4$ & 3.40 \\
\hline $\mathrm{Al}_{9}$ & $41.30-48.80$ & $\begin{array}{c}45.2 \\
6 \\
\end{array}$ & $\begin{array}{l}7.50- \\
11.30 \\
\end{array}$ & 9.02 & Light brown & $4-6$ & 3.86 \\
\hline $\mathrm{Al}_{10}$ & $41.30-48.80$ & $\begin{array}{c}44.5 \\
0 \\
\end{array}$ & $\begin{array}{l}7.50- \\
11.30\end{array}$ & 8.76 & Light brown & $3-5$ & 3.40 \\
\hline $\mathrm{Al}_{11}$ & $75.00-90.00$ & $\begin{array}{c}77.8 \\
3\end{array}$ & $\begin{array}{l}7.50- \\
11.30\end{array}$ & 8.26 & Dark brown & $6-8$ & 6.80 \\
\hline $\mathrm{Al}_{12}$ & $75.00-90.00$ & $\begin{array}{c}81.5 \\
0\end{array}$ & $\begin{array}{l}7.50- \\
11.30\end{array}$ & 8.51 & Dark brown & $7-9$ & 7.53 \\
\hline S.Em \pm & & 0.30 & & 0.44 & & & 0.45 \\
\hline $\begin{array}{l}\text { C.D.at } \\
1 \% \\
\end{array}$ & & 0.87 & & 1.23 & & & 0.61 \\
\hline
\end{tabular}

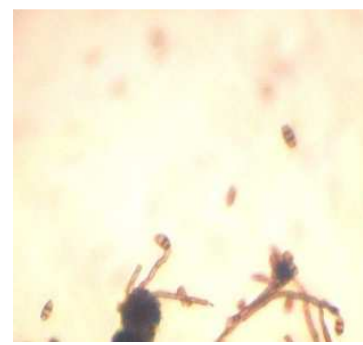

Patna isolate $\left(\mathrm{Al}_{1}\right)$

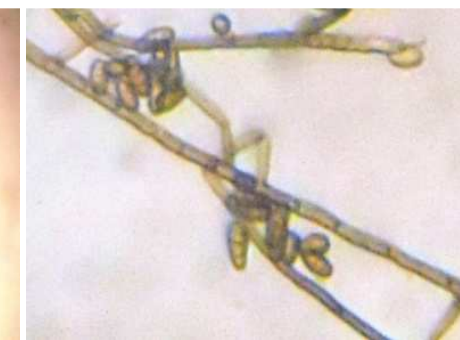

Bilaspur isolate $\left(\mathrm{Al}_{2}\right)$

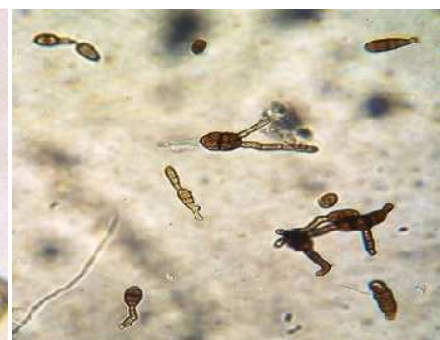

Sultanpur isolate $\left(\mathrm{Al}_{3}\right)$

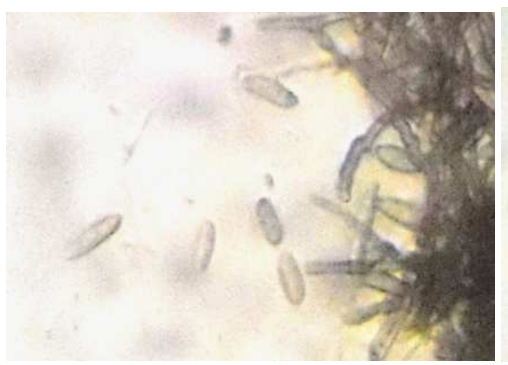

Mauranipur isolate $\left(\mathrm{Al}_{4}\right)$ Mashodha isolate $\left(\mathrm{Al}_{5}\right)$

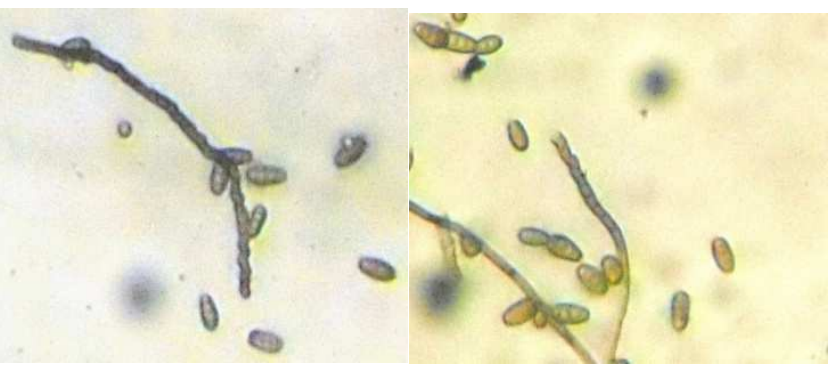

Kanpur isolate $\left(\mathrm{Al}_{6}\right)$

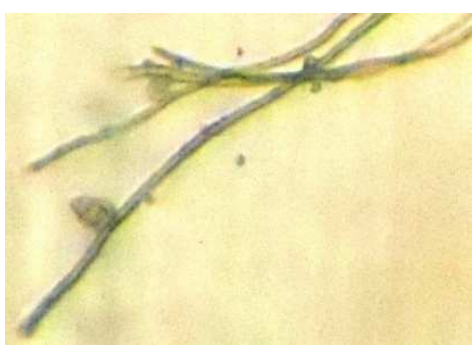

Kangra isolate $\left(\mathrm{Al}_{7}\right)$

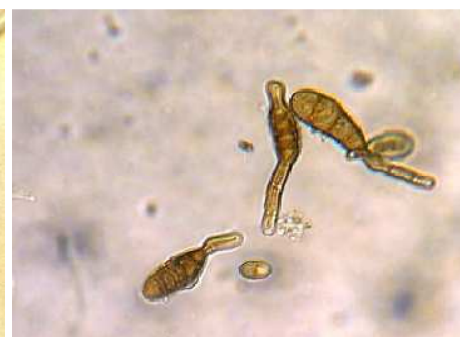

Ranchi isolate $\left(\mathrm{Al}_{8}\right)$

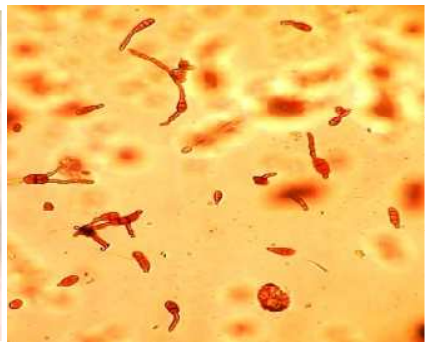

Mirzapur isolate ( $\mathrm{Al}_{9}$ ) 


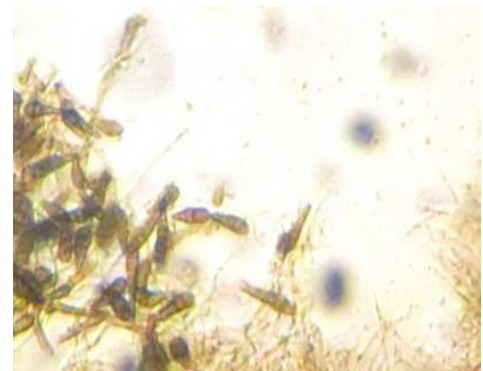

Gorakhpur Isolate $\left(\mathrm{Al}_{10}\right)$

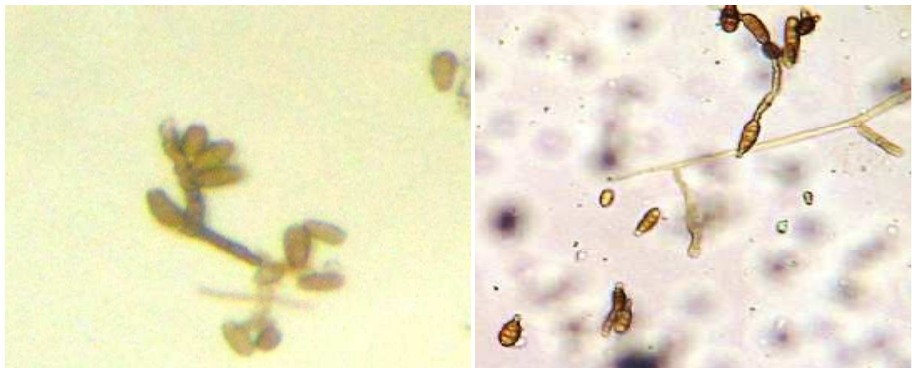

Berhampur Isolate $\left(\mathrm{Al}_{11}\right) \quad$ Kumarganj isolate $\left(\mathrm{Al}_{12}\right)$

Figure 7: Morphological Characters of Isolates $\left(\mathbf{A l}_{1}, \mathbf{A l}_{2}, \mathbf{A l}_{3}, \mathbf{A l}_{4}, \mathbf{A l}_{5}, \mathbf{A l}_{6}, \mathbf{A l}_{7}, \mathbf{A l}_{8}, \mathbf{A l}_{9}, \mathbf{A l}_{10}, \mathbf{A l}_{11} \mathbf{A n d}_{\mathbf{A l}}\right)$

\section{CONCLUSIONS}

The diseased samples were collected from thirteen places of India. Out of thirteen samples, pathogen causing disease could be isolated successfully from 12 samples and one obtained from Jammu (J\&K) could not be isolated. These isolates were purified by single spore technique method and identified as Alternaria lini. All the isolates were cultured separately on different media and showed variable reaction in respect of colonies colour, appearance, growth pattern, shape of colonies, margin, zonation, sporulation and radial growth. On all the test media the colonies colours of different isolates were observed as whitish, dirty white, light brown and dark brown. Radial growths of colonies formed by different isolates of A.lini were recorded after 7 days of inoculation found variable. Isolates $\mathrm{Al}_{11}$ collected from Berhampur (W.B.) showed maximum radial growth and isolates $\mathrm{Al}_{6}$ obtained from Kanpur (U.P.) showed minimum radial growth on all the test media. Shape of colonies formed by all the isolates on each media was circular. Colonies growths were varied from slow to fast on different culture media in case of all the isolates. Sporulation in different isolates on different culture media was also found variable. Some isolates showed early sporulation, some medium and some late on different media. Appearances of colonies formed on different media by different isolates were cottony, feathery, fluffy or compressed $\&$ thin. Average length of conidia of $A$. lini obtained from different isolates, ranged between $23.26 \mu \mathrm{m}$ ( $\mathrm{Al}_{7}$ of Kangra) to $45.72 \mu \mathrm{m}\left(\mathrm{Al}_{11}\right.$ of Berhampur) and width between $6.67 \mu \mathrm{m}$ ( $\mathrm{Al}_{4}$ of Mauranipur) to $17.01 \mu \mathrm{m}\left(\mathrm{Al}_{11}\right.$ of Berhampur). Average number of horizontal septa, in conidia of different isolates ranged between 1.86 ( $\mathrm{Al}_{8}$ of Ranchi) to 5.60 ( $\mathrm{Al}_{11}$ of Berhampur). Most of conidia obtained from different isolates were found light brown and few were dark brown in colour. Shape of conidia were also found variable and noted as oval, obclavate and clavate type. The conidia obtained from most of the isolates on PDA were unbeaked $\left(\mathrm{Al}_{1}, \mathrm{Al}_{2}, \mathrm{Al}_{4}, \mathrm{Al}_{5}, \mathrm{Al}_{6}, \mathrm{Al}_{7}, \mathrm{Al}_{8}, \mathrm{Al}_{10}\right)$, some were with rudimentary beaked $\left(\mathrm{Al}_{11}\right.$ and $\left.\mathrm{Al}_{12}\right)$ and few were short beaked $\left(\mathrm{Al}_{3}\right.$ and $\left.\mathrm{Al}_{9}\right)$. Morphological characters of conidiophores were also found variable in respect of length (33.00 to $81.50 \mu \mathrm{m}$ ), width (4.54 to $9.27 \mu \mathrm{m}$ ), colour (light brown to dark brown) and number of septa (3.33 to 7.53 ).

\section{REFERENCES}

1. Anonymous (2013).Annual Progress Report 2013-14. All India Coordinated Research Project on oilseeds (linseed). Directorate of Oilseed Research, Rajendranagar, Hyderabad, pp. 177.

2. Goyal, P., Chahar, M., Mathur, A.P., Kumar, A. and Chattopadhyay, C. (2011). Morphological andcultural variation in different oilseed Brassica isolates of Alternariabrassicaefrom different geographical regions of India. Indian Journal of Agricultural Sciences. 81 (11): 1052-1058.

3. Khulbe, A., Awasthi, R.P. and Tewari, A.K. (2011).Morphological and cultural diversity in isolates of Alternariabrassicae infecting rapeseed and mustard.Pantnagar Journal of Research.9 (2): 206-209. 
4. Kolte, S.J. and Fitt, B.D.L. (1997). “Disease of Linseed and Fibre Flax.”Shipra Publications, Delhi, pp. 247.

5. Kumar,V., Haldar,S., Pandey, K.K.,Singh, R.P., Singh, A.K. and Singh, P.C. (2007). Cultural, morphological,pathogenic and molecular variability amongst tomato isolates of Alternariasolani in India. World Journal of Microbiology and Biotechnology.24: 1003-1009.

6. Akash Verma, Sobita Simon, Kamaluddeen \& Yojna Lal, Efficacy of Indigenous Products and Carbendazim on Alternaria Blight [(Alternaria Lini) Dey] of Linseed (Linum Usitatissimum L.) Under Allahabad Conditions, International Journal of Agricultural Science and Research (IJASR), Volume 3, Issue 5, November - December 2013, pp. 33-38

7. Meena, P.D., Chattopadhyay, C., Kumar, V.R., Meena, R.L. and Rana, U.S. (2005). Spore behavior in atmosphere and trends in variability of AlternariabrassicaePopulation in India.Journal of Mycology and Plant Pathology.35 (3): 511.

8. Pramila.,Giri,K., Tasleem, M., Taj,G., Mal,R. and Kumar,A. (2014). Morphological, cultural, pathogenic and molecular variability amongst Indian mustard isolates of Alternariabrassicae in Uttarakhand. African Journal of Biotechnology.13(3): 441-448.

9. Sharma, M., Deep, S., Bhati, D. S. Chowdappa, P., Selvamani, R. and Sharma, P. (2013). Morphological, cultural, pathogenic and molecular studies of Alternaria brassicae infecting cauliflower and mustard in India. African Journal of Microbiology Research.7 (26):3351-3363.

10. Singh, R. B. and Singh, R. N. (2004).Management of alternaria blight of linseed. Annals of Plant Protection Sciences.12(2): 305-309.

11. Singh,A., Singh,V. and Yadav,S.M. (2014). Cultural, morphological and pathogenic variability of Alternariasolani causing early blight in tomato.Science Alert.13: 167-172.

12. Vajpeyi, M., Dubey, S.D. and Srivastava, R.L. (2005).Linseed : Industrial and Medicinal uses. AICRP on linseed, P.C. Unit (linseed) Kanpur.pp.16 